\title{
Boundary controlled irreversible port-Hamiltonian systems
}

\author{
Hector Ramirez ${ }^{\mathrm{a}}$, Yann Le Gorrec ${ }^{\mathrm{b}}$, Bernhard Maschke $\mathrm{c}^{\mathrm{c}}$ \\ ${ }^{a}$ Universidad Técnica Federico Santa María, Valparaiso, Chile. (hector.ramireze@usm.cl). \\ ${ }^{b}$ Département d'Automatique et Systèmes Micro-Mécatroniques, FEMTO-ST UMR CNRS 6174, Université de Bourgogne Franche Comté, 26 \\ chemin de l'épitaphe, F-25030 Besançon, France. (legorrec@femto-st.fr). \\ ${ }^{c}$ Laboratoire d'Automatique et Génie des Procédés CNRS UMR 5007, Université de Lyon, Université Lyon 1, F-69622 Villeurbanne, France \\ (maschke@lagep.univ-lyon1.fr)
}

\begin{abstract}
Boundary controlled irreversible port-Hamiltonian systems (BC-IPHS) on 1-dimensional spatial domains are defined by extending the formulation of reversible BC-PHS to irreversible thermodynamic systems controlled at the boundaries of their spatial domains. The structure of BC-IPHS has clear physical interpretation, characterizing the coupling between energy storing and energy dissipating elements. By extending the definition of boundary port variables of BC-PHS to deal with the dissipative terms, a set of boundary port variables are defined such that BC-IPHS are passive with respect to a given set of conjugated inputs and outputs. As for finite dimensional IPHS, the first and second principle are satisfied as a structural property. Several examples are given to illustrate the proposed approach.
\end{abstract}

Keywords: Port-Hamiltonian systems, irreversible thermodynamics, infinite dimensional systems

\section{Introduction}

The control of processes in Chemical Engineering is a highly difficult problem due to the nonlinearities induced as well by their thermodynamic properties as their flux relations. One very fruitful approach for the synthesis of nonlinear controllers is to use the properties of the dynamical models arising from first principle modeling such as symmetries, invariants and more generally balance equations of particular thermodynamic potential functions such as the entropy. These balance equations may be used as dissipation inequalities in passivity-based control as introduced in Willems (1972) and is now a well-developed branch of control van der Schaft, 2000; Brogliato et al., 2020).

In the case of chemical engineering processes, various thermodynamic potentials, such as the entropy or Helmholtz free energy, may be used as storage functions in a dissipation inequality Alonso and Ydstie, 1996) and may be used for control design methods based on Lyapunov control functions Christofides and Daoutidis, 1998; Christofides, 2001) and passivity (Alonso and Ydstie, 1996, 2001; Alonso et al., 2002; (Schaum et al., 2018).

The derivation of these Lyapunov functions and control Lyapunov functions are in most cases, based on the axioms of Equilibrium and Irreversible Thermo- dynamics and the structure of the dynamical models for these systems. A variety of such "thermodynamic" dynamical models have been suggested which are generalization of gradient control systems Cortés et al. 2005), Lagrangian control systems (Ortega et al. 1998), Hamiltonian control systems (Brockett, 1977; van der Schaft, 1986; Nijmeijer and van der Schaft, 1990), (Marsden, 1992, chap. 7) or Port Hamiltonian systems (Maschke and van der Schaft, 1992; van der Schaft and Maschke, 1995; Duindam et al., 2009; van der Schaft and Jeltsema, 2014) in the sense that they should account both for the conservation of the total energy and for the irreversible entropy production.

A first class of these thermodynamic control systems is defined by pseudo-gradient systems (Favache and Dochain, 2010; Favache et al., 2011), meaning that they are redefined with respect to a pseudo-metric, in a very similar way as suggested for electrical circuits in Brayton and Moser (1964); Smale (1972). A second class of systems is defined as metriplectic systems (sum of Hamiltonian and gradient systems) with one or two generating functions (Grmela and Öttinger, 1997; Öttinger and Grmela, 1997; Mushik et al., 2000; Ramirez. et al., 2009; Hoang et al., 2011, 2012). A third class of systems is defined as nonlinearly constrained Lagrangian systems (Gay-Balmaz and Yoshimura, 2018). A fourth 
class of systems is defined as implicit Hamiltonian control systems, in the sense that they are defined on a submanifold of some embedding space (the Thermodynamic Phase space or its symplectic extension), by control Hamiltonian systems defined on contact manifolds (Mrugala et al., 1991; Eberard et al., 2007; Favache et al., 2010; Ramirez et al., 2013b, a, 2017a; Merker and Krüger, 2013) or their symplectization van der Schaft and Maschke, 2018).

In this paper we shall be interested in another class, namely the Irreversible port-Hamiltonian System (IPHS) which were suggested in (Ramirez et al., $2013 \mathrm{~b}$ ) as an extension of port-Hamiltonian systems using a quasi-Poisson bracket and still embedding the irreversible entropy creation. For processes described by lumped models it has been shown that the formalism encompasses a large and general class of irreversible thermodynamic systems, such as heat-exchangers, chemical reactions, chemical reaction networks and coupled mechanic-thermodynamic systems (Ramirez et al., 2013c). Moreover, using the definition of the availability function Keenan, 1951; Alonso and Ydstie, 2001) the IPHS structure has recently been employed to exploit the thermodynamic properties of irreversible processes to derive non-linear passivity based controllers (Ramirez et al., 2016).

This paper extends the IPHS formulation to systems defined on 1-dimensional spatial domains building on the framework of boundary controlled PHS van der Schaft and Maschke, 2002) using the formulation on 1D spaces (Le Gorrec et al., 2005; Jacob and Zwart, 2012) with the aim of using the passivity-based and geometric control design methods, such as methods based on invariance (Godasi et al., 2002), Casimir functions (Macchelli et al., 2017; Macchelli et al., 2020) or (non)-linear dynamic boundary control (Ramirez et al., 2014a, 2017b). Note that a first approach in this line was given in (Ramirez and Le Gorrec, 2016) for a diffusion process.

The paper is organized as follows. In Section 2 a 1-D isentropic fluid, with and without dissipation, is presented as motivating example to contextualize the proposed irreversible model. Section 3 presents the main contribution of the paper, namely the definition of boundary controlled IPHS on a 1D spatial domain. Section 4 gives two important lemmas regarding the passivity of the system, namely the energy conservation and the irreversible entropy production. In Section 5 the IPHS model of a general diffusion-reaction process is presented, and finally in Section 6 some conclusions and comments on future work are given. An Appendix with the basic definitions of boundary controlled PHS is also included.

\section{Motivating example: 1-D compressible fluid}

\subsection{The isentropic fluid: the reversible case}

Let us first consider the dynamic behavior of an 1-D isentropic fluid in Lagrangian coordinates, also known as $p$-system, and recall its Port Hamiltonian formulation (Maschke and van der Schaft, 2005). The 1-D spatial domain is the interval $[a, b] \ni z, a, b \in \mathbb{R}, a<b$. Using as state variables the specific volume $\phi(t, z)$ and the velocity $v(t, z)$ of the fluid, the dynamical model of the fluid is given by the mass balance equation (expressed in terms of the specific volume) and the momentum balance equation (expressed in terms of the velocity seen as "momentum density")

$$
\begin{aligned}
& \frac{\partial \phi}{\partial t}(t, z)=\frac{\partial v}{\partial z}(t, z) \\
& \frac{\partial v}{\partial t}(t, z)=-\frac{\partial p}{\partial z}(t, z)
\end{aligned}
$$

where $p(\phi)$ is the pressure of the fluid. The Hamiltonian formulation is obtained by considering the total energy of the system which consists in the sum of the kinetic and the internal energy, denoting the internal energy density by $u(\phi)$

$$
H(v, \phi)=\int_{a}^{b}\left(\frac{1}{2} v^{2}+u(\phi)\right) d z
$$

The variational derivative of the total energy yields $\frac{\delta H}{\delta v}=$ $v$ and $\frac{\delta H}{\delta \phi}=\frac{\partial u}{\partial \phi}=-p$ and the system (1)-(2) may be written as the Hamiltonian system

$$
\left[\begin{array}{l}
\frac{\partial \phi}{\partial t} \\
\frac{\partial v}{\partial t}
\end{array}\right]=P_{1} \frac{\partial}{\partial z}\left(\left[\begin{array}{l}
\frac{\delta H}{\delta \phi} \\
\frac{\delta H}{\delta v}
\end{array}\right]\right)
$$

where $P_{1}=\left[\begin{array}{ll}0 & 1 \\ 1 & 0\end{array}\right]$ and $P_{1} \frac{\partial}{\partial z}$ is a Hamiltonian operator (Olver, 1993; van der Schaft and Maschke, 2002). Considering an open system, when there is mass and energy flow through the boundary (at the points $a$ and $b$ ), the Hamiltonian system (3) is completed with a pair of boundary port input and output $(v, y)$ as follows

$$
\left[\begin{array}{l}
v \\
y
\end{array}\right]=\left[\begin{array}{l}
W_{B} \\
W_{C}
\end{array}\right]\left[\begin{array}{c}
\frac{\delta H}{\delta \phi}(b) \\
\frac{\delta H}{\delta v}(b) \\
\frac{\delta H}{\delta \phi}(a) \\
\frac{\delta H}{\delta v}(a)
\end{array}\right]=\left[\begin{array}{cccc}
1 & 0 & 0 & 0 \\
0 & 0 & -1 & 0 \\
0 & 1 & 0 & 0 \\
0 & 0 & 0 & 1
\end{array}\right]\left[\begin{array}{c}
-p(t, b) \\
v(t, b) \\
-p(t, a) \\
v(t, a)
\end{array}\right]
$$

from where $v(t)=\left[\begin{array}{c}-p(t, b) \\ p(t, a)\end{array}\right]$ and $y(t)=\left[\begin{array}{l}v(t, b) \\ v(t, a)\end{array}\right]$. It is direct to verify that this choice of inputs and outputs satisfies A.2 , hence the change of energy of the system is given by $\dot{H}(t)=y^{\top}(t) v(t)$. 


\subsection{The isentropic fluid: the irreversible case}

Consider that there is dissipation in the system given by viscous damping. The balance equations are then given by

$$
\begin{aligned}
& \frac{\partial \phi}{\partial t}(t, z)=\frac{\partial v}{\partial z}(t, z) \\
& \frac{\partial v}{\partial t}(t, z)=-\frac{\partial p}{\partial z}(t, z)-\frac{\partial \tau}{\partial z}(t, z)
\end{aligned}
$$

where $\tau$ is the viscous tensor defined as $\tau=-\hat{\mu} \frac{\partial v}{\partial z}$, with $\hat{\mu}$ the viscous damping coefficient. The system contains dissipation or rather a irreversible phenomenon induced by the viscosity of the fluid. Therefore we account for the thermal domain and consider Gibbs' equation $d u=$ $-p d \phi+T d s$ where $s$ denotes the entropy density and $T$ the temperature. The total energy of the system is still the sum of the kinetic and the internal energy

$$
H(v, \phi, s)=\int_{a}^{b}\left(\frac{1}{2} v^{2}+u(\phi, s)\right) d z
$$

The mass balance (4) and momentum balance equations (5) are then augmented with the entropy balance equation

$$
\frac{\partial s}{\partial t}(t, z)=\frac{\hat{\mu}}{T}\left(\frac{\partial v}{\partial z}\right)^{2}(t, z)
$$

and the system of balance equations may be written as the quasi-Hamiltonian system

$$
\left[\begin{array}{l}
\frac{\partial \phi}{\partial t} \\
\frac{\partial v}{\partial t} \\
\frac{\partial s}{\partial t}
\end{array}\right]=\left[\begin{array}{ccc}
0 & \frac{\partial(\cdot)}{\partial z} & 0 \\
\frac{\partial(\cdot)}{\partial z} & 0 & \frac{\partial}{\partial z}\left(\frac{\hat{\mu}}{T}\left(\frac{\partial v}{\partial z}\right)(\cdot)\right) \\
0 & \frac{\hat{\mu}}{T}\left(\frac{\partial v}{\partial z}\right) \frac{\partial(\cdot)}{\partial z} & 0
\end{array}\right]\left(\left[\begin{array}{c}
\frac{\delta H}{\delta \phi} \\
\frac{\delta H}{\delta v} \\
\frac{\delta H}{\delta s}
\end{array}\right]\right)
$$

As the operator depends on the co-energy variable $T$, it is only a quasi-Hamiltonian operator.

In the following section this latter formulation will be used to define boundary controlled irreversible portHamiltonian systems, extending the framework originally proposed in Ramirez et al. (2013b) for irreversible thermodynamic systems on finite dimensional spaces to systems defined on infinite dimensional spaces.

\section{Boundary controlled IPHS}

In this section, we introduce the Irreversible Boundary Port Hamiltonian System (IPHS) defined on a 1D spatial domain $z \in[a, b], a, b \in \mathbb{R}, a<b$. The state variables of the system are the $n+1$ extensive variables 1 .

\footnotetext{
${ }^{1} \mathrm{~A}$ variable is qualified as extensive when it characterizes the thermodynamic state of the system and its total value is given by the sum of its constituting parts.
}

The following partition of the state vector shall be considered: the first $n$ variables by $x=\left[q_{1}, \ldots, q_{n}\right]^{\top} \in \mathbb{R}^{n}$ and the entropy density by $s \in \mathbb{R}$. The thermodynamic properties of the system are expressed by Gibbs' equation (Callen, 1985), which we give here in its local form with pairs of specific energy-conjugated variables (Duindam et al., 2009, Chapter 3)

$$
d h=T d s+p_{i} \sum_{i=1}^{n} d q_{i}
$$

where $T$ is the temperature, conjugated to the entropy density, and the variables $p_{i}$ denote the intensive variables, which are conjugated to the $q_{i}$ variables. Gibbs' equation is here understood in a general context in order to account for coupled thermoelectro/magnetic/mechanical systems. Gibbs' equation is equivalent to the existence of an energy functional

$$
H(x, s)=\int_{a}^{b} h(x(z), s(z)) d z
$$

where $h(x, s)$ is the energy density function. The total entropy functional is denoted by

$$
S(t)=\int_{a}^{b} s(z, t) d z
$$

The following pseudo (locally defined) brackets will be used to define the thermodynamic driving forces of the system

$$
\begin{aligned}
\{\Gamma|\mathcal{G}| \Omega\} & =\left[\begin{array}{l}
\frac{\delta \Gamma}{\delta x} \\
\frac{\delta \Gamma}{\delta s}
\end{array}\right]\left[\begin{array}{cc}
0 & \mathcal{G} \\
-\mathcal{G}^{*} & 0
\end{array}\right]\left[\begin{array}{c}
\frac{\delta \Omega}{\delta x} \\
\frac{\delta \Omega}{\delta s}
\end{array}\right], \\
\{\Gamma \mid \Omega\} & =\frac{\delta \Gamma^{\top}}{\delta s}\left(\frac{\partial}{\partial z} \frac{\delta \Omega}{\delta s}\right)
\end{aligned}
$$

for some smooth functions $\Gamma, \Omega$ and $\mathcal{G}$.

We shall first define a system of balance equations in terms of an Irreversible (quasi-)Hamiltonian system.

Definition 1. An infinite dimensional IPHS undergoing $m$ irreversible processes is defined by the PDE

$$
\begin{aligned}
& \frac{\partial}{\partial t}\left[\begin{array}{c}
x(t, z) \\
s(t, z)
\end{array}\right]=\left[\begin{array}{cc}
P_{0} & G_{0} \mathbf{R}_{\mathbf{0}}(\mathbf{x}) \\
-\mathbf{R}_{\mathbf{0}}(\mathbf{x})^{\top} G_{0}^{\top} & 0
\end{array}\right]\left[\begin{array}{l}
\frac{\delta H}{\delta x}(t, z) \\
\frac{\delta H}{\delta s}(t, z)
\end{array}\right]+ \\
& {\left[\begin{array}{cc}
P_{1} \frac{\partial(.)}{\partial z} & \frac{\partial\left(G_{1} \mathbf{R}_{\mathbf{1}}(\mathbf{x}) .\right)}{\partial z} \\
\mathbf{R}_{\mathbf{1}}(\mathbf{x})^{\top} G_{1}^{\top} \frac{\partial(.)}{\partial z} & g_{s} \mathbf{r}_{\mathbf{s}}(\mathbf{x}) \frac{\partial(.)}{\partial z}+\frac{\partial\left(g_{s} \mathbf{r}_{\mathbf{s}}(\mathbf{x}) \cdot\right)}{\partial z}
\end{array}\right]\left[\begin{array}{l}
\frac{\partial H}{\delta x}(t, z) \\
\frac{\delta H}{\delta s}(t, z)
\end{array}\right]}
\end{aligned}
$$

where $P_{0}=-P_{0}^{\top} \in \mathbb{R}^{n \times n}, P_{1}=P_{1}^{\top} \in \mathbb{R}^{n \times n}, g_{s} \in \mathbb{R}, G_{0} \in$ $\mathbb{R}^{n \times m}, G_{1} \in \mathbb{R}^{n \times m}$ with $m$ the number of states involved in the entropy production. $\mathbf{R}_{\mathbf{0}} \in \mathbb{R}^{m \times 1}, \mathbf{R}_{\mathbf{1}} \in \mathbb{R}^{m \times 1}$ and 
$r_{s} \in \mathbb{R}$ stand for the vectors of modulated driving forces with

$$
\begin{gathered}
R_{0, i}=\gamma_{0, i}\left(x, z, \frac{\delta H}{\delta x}\right)\left\{S\left|G_{0}(:, i)\right| H\right\} \\
R_{1, i}=\gamma_{1, i}\left(x, z, \frac{\delta H}{\delta x}\right)\left\{S\left|G_{1}(:, i) \frac{\partial}{\partial z}\right| H\right\}
\end{gathered}
$$

and

$$
r_{s}=\gamma_{s}\left(x, z, \frac{\delta H}{\delta x}\right)\{S \mid H\}
$$

with $\gamma_{k, i}\left(x, z, \frac{\delta H}{\delta x}\right), \gamma_{s}\left(x, z, \frac{\delta H}{\delta x}\right): \mathbb{R}^{n} \rightarrow \mathbb{R}, \gamma_{k, i}, \gamma_{s} \geq 0$, non-linear positive functions.

Remark 2. Definition $\square$ is an extension of the definition of IPHS for finite dimensional systems presented in Ramirez et al. (2013b.c) for thermodynamic systems defined on $1 D$ spatial domains. In fact it is not difficult to verify that if finite dimensional system is considered only the zero order operator matrix is present in (7) and Definition 1 reduces to

$$
\frac{d}{d t}\left[\begin{array}{c}
x(t, z) \\
s(t, z)
\end{array}\right]=\left[\begin{array}{cc}
P_{0} & G_{0} \mathbf{R}_{\mathbf{0}}(\mathbf{x}) \\
-\mathbf{R}_{\mathbf{0}}(\mathbf{x})^{\top} G_{0}^{\top} & 0
\end{array}\right]\left[\begin{array}{l}
\frac{\delta H}{\delta x}(t, z) \\
\frac{\delta H}{\delta s}(t, z)
\end{array}\right]
$$

which is equivalent to the definition in Ramirez et al. (2013b c) for the case $m=1$ or Ramirez et al. (2014b, 2016) for $m>1$.

Remark 3. The pseudo-brackets (6) define the thermodynamic driving forces of the process. Notice that when considering a finite dimensional system (6) is equal to the pseudo-bracket defined in Ramirez et al. (2013b.c) for lumped IPHS.

In the following definition the above system is completed with boundary port variables.

Definition 4. A boundary controlled IPHS (BC-IPHS) is an infinite dimensional IPHS according to Definition 1 equipped with boundary inputs and outputs, defined as the linear combinations of the boundary port variables, respectively

$$
v(t)=W_{B}\left[\begin{array}{l}
e(t, b) \\
e(t, a)
\end{array}\right], \quad y(t)=W_{C}\left[\begin{array}{l}
e(t, b) \\
e(t, a)
\end{array}\right]
$$

where the boundary port variables are

$$
e(t, z)=\left[\begin{array}{c}
\frac{\delta H}{\delta x}(t, z) \\
\mathbf{R}(\mathbf{x}) \frac{\delta H}{\delta s}(t, z)
\end{array}\right]
$$

$$
\begin{aligned}
& \text { with } \mathbf{R}(\mathbf{x})=\left[\begin{array}{lll}
1 & \mathbf{R}_{\mathbf{1}}(\mathbf{x}) & \mathbf{r}_{\mathbf{s}}(\mathbf{x})
\end{array}\right]^{\top} \text { and } \\
& \qquad \begin{array}{ll}
W_{B}=\left[\begin{array}{ll}
\frac{1}{\sqrt{2}}\left(\Xi_{2}+\Xi_{1} P_{e p}\right) M_{p} & \frac{1}{\sqrt{2}}\left(\Xi_{2}-\Xi_{1} P_{e p}\right) M_{p}
\end{array}\right], \\
W_{C}=\left[\begin{array}{ll}
\frac{1}{\sqrt{2}}\left(\Xi_{1}+\Xi_{2} P_{e p}\right) M_{p} & \frac{1}{\sqrt{2}}\left(\Xi_{1}-\Xi_{2} P_{e p}\right) M_{p}
\end{array}\right],
\end{array}
\end{aligned}
$$

where $M_{p}=\left(M^{\top} M\right)^{-1} M^{\top}, P_{e p}=M^{\top} P_{e} M$ and $M$ is spanning the columns of $P_{e}$, defined $b y{ }^{2}$

$$
P_{e}=\left[\begin{array}{cccc}
P_{1} & 0 & G_{1} & 0 \\
0 & 0 & 0 & g_{s} \\
G_{1}^{\top} & 0 & 0 & 0 \\
0 & g_{s} & 0 & 0
\end{array}\right]
$$

and where $\Xi_{1}$ and $\Xi_{2}$ satisfy $\Xi_{2}^{\top} \Xi_{1}+\Xi_{1}^{\top} \Xi_{2}=0$ and $\Xi_{2}^{\top} \Xi_{2}+\Xi_{1}^{\top} \Xi_{1}=I$

Remark 5. Definition 4 proposes an extension of the boundary port variables defined in Le Gorrec et al. (2005) for (reversible) BC-PHS to deal with nonreversible systems. Indeed, the vector of boundary port-variables (8) is composed by the vector of intensive variables and the vector of modulated driving forces. On the other hand the matrix (9), which is defined by the reversible structure matrix $P_{1}$ and the irreversible structure matrices $G_{1}$ and $g_{s}$, define the admissible parametrization to obtain the boundary inputs and outputs. Notice that when no irreversible phenomena is present, and thus the entropy coordinate is not considered, Definition 11 and 4 define a BC-PHS (Le Gorrec et al., 2005) (see also the Appendix A.).

Example 6. Recalling the $1 D$ fluid model in Section 2. its BC-IPHS formulation is given by $P_{0}=0, G_{0}=$ $0, g_{s}=0, P_{1}=\left[\begin{array}{ll}0 & 1 \\ 1 & 0\end{array}\right]$ and $G_{1}=\left[\begin{array}{l}0 \\ 1\end{array}\right]$ with $x=\left[\begin{array}{l}\phi \\ v\end{array}\right]$ and $R_{11}=\gamma_{1}\left\{S\left|G_{1}(:, 1) \frac{\partial}{\partial z}\right| H\right\}$ with $\gamma_{1}=\frac{\hat{\mu}}{T}>0$. In this case $n=2, m=1$ and

$$
P_{e}=\left[\begin{array}{lllll}
0 & 1 & 0 & 0 & 0 \\
1 & 0 & 0 & 1 & 0 \\
0 & 0 & 0 & 0 & 0 \\
0 & 1 & 0 & 0 & 0 \\
0 & 0 & 0 & 0 & 0
\end{array}\right]
$$

which gives $M=\left[\begin{array}{ccccc}\frac{1}{2} & 0 & 0 & \frac{1}{2} & 0 \\ 0 & 1 & 0 & 0 & 0\end{array}\right]^{\top}, \quad M_{P}=$ $\left[\begin{array}{ccccc}0 & 1 & 0 & 0 & 0 \\ 1 & 0 & 0 & 1 & 0\end{array}\right]$ and $P_{e p}=\left[\begin{array}{ll}0 & 1 \\ 1 & 0\end{array}\right]$. Choosing the
parametrization

$$
\Xi_{1}=\frac{1}{\sqrt{2}}\left[\begin{array}{ll}
1 & 0 \\
1 & 0
\end{array}\right], \quad \Xi_{2}=\frac{1}{\sqrt{2}}\left[\begin{array}{cc}
0 & 1 \\
0 & -1
\end{array}\right]
$$

define the following boundary inputs and outputs

$$
v(t)=\left[\begin{array}{c}
-p(t, b)+\frac{\hat{\mu}}{T} \frac{\partial v}{\partial z}(t, b) \\
p(t, a)-\frac{\hat{\mu}}{T} \frac{\partial v}{\partial z}(t, a)
\end{array}\right], \quad y(t)=\left[\begin{array}{c}
v(t, b) \\
v(t, a)
\end{array}\right] .
$$

\footnotetext{
${ }^{2} 0$ has to be understood as the zero matrix of proper dimensions.
} 
As for the reversible case, the boundary inputs and outputs correspond, respectively, to the pressure and the velocities evaluated at points $a$ and $b$. If there is no dissipation in the system, then the boundary inputs and outputs are exactly the same as for the reversible case. It is direct to verify that the internal energy balance is given by $\dot{H}(t)=y(t)^{\top} v(t)$. Notice that the chosen parametrization is not unique and that a different choice will lead to different boundary inputs/outputs.

Example 7. Consider the heat conduction with heat diffusion over a $1 D$ spatial domain. The conserved quantity is the density of internal energy and the state reduces to a unique variable. Choose the internal energy density $u=u(s)$ as thermodynamic potential function (and $U(s)=\int_{a}^{b} u d z$ ), in this case Gibbs relation defines the temperature as intensive variable conjugated to the extensive variable, the entropy by $T=\frac{d u}{d s}(s)$. This leads to write the following entropy balance equation (Duindam et al., 2009)

$$
\frac{\partial s}{\partial t}=-\frac{1}{T} \frac{\partial}{\partial z}\left(-\lambda \frac{\partial T}{\partial z}\right)
$$

where $\lambda$ denotes the heat conduction coefficient and $-\lambda \frac{\partial T}{\partial z}=f_{Q}$ corresponds to the heat flux. Alternatively the heat conduction can be written in terms of the entropy flux $f_{S}=\frac{1}{T} f_{Q}=-\frac{\lambda}{T} \frac{\partial T}{\partial z}$,

$$
\frac{\partial s}{\partial t}=\frac{\partial}{\partial z}\left(\frac{\lambda}{T} \frac{\partial T}{\partial z}\right)+\frac{\lambda}{T^{2}}\left(\frac{\partial T}{\partial z}\right)^{2}
$$

from where the entropy production $\sigma_{s}=\frac{\lambda}{T^{2}}\left(\frac{\partial T}{\partial z}\right)^{2}$ is directly identified. Recalling that $\frac{\delta U}{\delta s}=T$, the IPHS formulation of the heat conduction is directly obtained from (10),

$$
\frac{\partial s}{\partial t}=\frac{\lambda}{T^{2}} \frac{\partial T}{\partial z} \frac{\partial}{\partial z}\left(\frac{\delta U}{\delta s}\right)+\frac{\partial}{\partial z}\left(\frac{\lambda}{T^{2}} \frac{\partial T}{\partial z}\left(\frac{\delta U}{\delta s}\right)\right)
$$

which is equivalent to (7) where $P_{0}=0, P_{1}=0, G_{0}=0$, $G_{1}=0, g_{s}=1$ and $r_{s}=\gamma_{s}\{S \mid U\}$ with $\gamma_{s}=\frac{\lambda}{T^{2}}$ and $\{S \mid U\}=\frac{\partial T}{\partial z}$. In this case $P_{e}=\frac{1}{2}\left[\begin{array}{ll}0 & 1 \\ 1 & 0\end{array}\right], n=1$ and $m=1$. Choosing $\Xi_{1}=\frac{1}{\sqrt{2}}\left[\begin{array}{ll}1 & 0 \\ 1 & 0\end{array}\right], \Xi_{2}=\frac{1}{\sqrt{2}}\left[\begin{array}{cc}0 & 1 \\ 0 & -1\end{array}\right]$ the boundary inputs and outputs of the system are

$$
v(t)=\left[\begin{array}{c}
\left(\frac{\lambda_{s}}{T} \frac{\partial T}{\partial z}\right)(t, b) \\
-\left(\frac{\lambda_{s}}{T} \frac{\partial T}{\partial z}\right)(t, a)
\end{array}\right], \quad y(t)=\left[\begin{array}{c}
T(t, b) \\
T(t, a)
\end{array}\right],
$$

respectively the entropy flux and the temperature at each boundary.

\section{Energy and entropy balance equations}

BC-IPHS encode the first and second principle of Thermodynamics, i.e., the conservation of the total energy and the irreversible production of entropy as stated in the following lemmas.

Lemma 8. (Conservation of energy) The total energy balance is

$$
\dot{H}=y(t)^{\top} v(t)
$$

which leads, when the input is set to zero, to $\dot{H}=0$ in accordance with the first principle of Thermodynamics.

Proof. The variation of the total energy with respect to time is

$$
\begin{aligned}
\dot{H} & =\int_{a}^{b} \frac{\partial h}{\partial t} d z=\int_{a}^{b}\left(\frac{\delta H^{\top}}{\delta x} \frac{\partial x}{\partial t}+\frac{\delta H}{\delta s}^{\top} \frac{\partial s}{\partial t}\right) d z \\
& =\int_{a}^{b}\left[\frac{\delta H}{\delta x}(t, z)^{\top} \frac{\delta H}{\delta s}(t, z)\right] \mathcal{J}_{e}\left[\begin{array}{l}
\frac{\delta H}{\delta x}(t, z) \\
\frac{\delta H}{\delta s}(t, z)
\end{array}\right] d z
\end{aligned}
$$

with

$$
\mathcal{J}_{e}=\left[\begin{array}{cc}
P_{1} \frac{\partial(.)}{\partial z} & \frac{\partial\left(G_{1} \mathbf{R}_{\mathbf{1}}(\mathbf{x}) \cdot\right)}{\partial z} \\
\mathbf{R}_{\mathbf{1}}(\mathbf{x})^{T} G_{1}^{T} \frac{\partial(.)}{\partial z} & g_{s} \mathbf{r}_{\mathbf{s}}(\mathbf{x}) \frac{\partial(.)}{\partial z}+\frac{\partial\left(g_{s} \mathbf{r}_{\mathbf{s}}(\mathbf{x}) \cdot\right)}{\partial z}
\end{array}\right]
$$

where we have used the skew symmetry of the matrix of zero order operators

$$
\left[\begin{array}{cc}
P_{0} & G_{0} \mathbf{R}_{\mathbf{0}}(\mathbf{x}) \\
-\mathbf{R}_{\mathbf{0}}(\mathbf{x})^{T} G_{0}^{T} & 0
\end{array}\right] .
$$

Noticing that

$$
\int_{a}^{b} \frac{\delta H^{\top}}{\delta x} P_{1} \frac{\partial}{\partial z}\left(\frac{\delta H}{\delta x}\right) d z=\frac{1}{2}\left[\frac{\delta H^{\top}}{\delta x} P_{1} \frac{\delta H}{\delta x}\right]_{a}^{b}
$$

that

$$
\begin{aligned}
\int_{a}^{b}\left(\frac{\delta H}{\delta s} \mathbf{R}_{\mathbf{1}}(\mathbf{x})^{T} G_{1}^{T} \frac{\partial}{\partial z}\left(\frac{\delta H}{\delta x}\right)\right. & \left.+\frac{\delta H^{\top}}{\delta x} \frac{\partial}{\partial z}\left(G_{1} \mathbf{R}_{\mathbf{1}}(\mathbf{x}) \frac{\delta H}{\delta s}\right)\right) d z \\
& =\left[\frac{\delta H}{\delta s} \mathbf{R}_{\mathbf{1}}(\mathbf{x})^{\top} G_{1}^{\top} \frac{\delta H}{\delta x}\right]_{a}^{b}
\end{aligned}
$$

and that

$$
\begin{array}{r}
\int_{a}^{b}\left(\frac{\delta H}{\delta s} g_{s} \mathbf{r}_{\mathbf{s}}(\mathbf{x}) \frac{\partial}{\partial z}\left(\frac{\delta H}{\delta s}\right)+\frac{\delta H}{\delta s} \frac{\partial}{\partial z}\left(g_{s} \mathbf{r}_{\mathbf{s}}(\mathbf{x}) \frac{\delta H}{\delta s}\right)\right) d z \\
=\left[\frac{\delta H}{\delta s} g_{s} \mathbf{r}_{\mathbf{s}}(\mathbf{x})\left(\frac{\delta H}{\delta s}\right)\right]_{a}^{b}
\end{array}
$$

we have

$$
\dot{H}=\left[\left[\begin{array}{c}
\frac{\delta H}{\delta x} \\
\frac{\delta H}{\delta s} \\
\mathbf{R}_{\mathbf{1}}(\mathbf{x}) G_{1}^{T} \frac{\delta H}{\delta s} \\
g_{s} \mathbf{r}_{\mathbf{s}}(\mathbf{x}) \frac{\delta H}{\delta s}
\end{array}\right]^{\top} P_{e}\left[\begin{array}{c}
\frac{\delta H}{\delta x} \\
\frac{\delta H}{\delta s} \\
\mathbf{R}_{\mathbf{1}}(\mathbf{x}) G_{1} \frac{\delta H}{\delta_{s}} \\
g_{s} \mathbf{r}_{\mathbf{s}}(\mathbf{x}) \frac{\delta H}{\delta s}
\end{array}\right]\right]_{a}^{b}
$$


with $P_{e}$ defined in (9). Using the parametrization proposed in (Le Gorrec et al., 2005; ; Le Gorrec et al., 2006) ( $P_{e}$ is potentially not full rank), it is possible to write

$$
\left[\begin{array}{l}
u(t) \\
y(t)
\end{array}\right]=\frac{1}{\sqrt{2}}\left[\begin{array}{ll}
\Xi_{1} & \Xi_{2} \\
\Xi_{2} & \Xi_{1}
\end{array}\right]\left[\begin{array}{cc}
P_{1 p} M_{p} & -P_{1 p} M_{p} \\
M_{p} & M_{p}
\end{array}\right]\left[\begin{array}{l}
e(t, b) \\
e(t, a)
\end{array}\right]
$$

with $\Xi_{i}, M_{P}$ and $P_{1 p}$ defined in Definition 4 from where it is obtained that $\dot{H}=y(t)^{\top} u(t)$.

Lemma 9. (Irreversible entropy production) The total entropy balance is given by

$$
\dot{S}=\int_{a}^{b} \sigma_{t} d z-y_{S}^{\top} v_{S}
$$

where $y_{s}$ and $v_{s}$ are the entropy conjugated input/output and $\sigma_{t}$ is the total internal entropy production. This leads, when the input is set to zero, to $\dot{S}=\int_{a}^{b} \sigma_{t} d z \geq 0$ in accordance with the second principle of Thermodynamics.

Proof. Let's consider the total entropy balance

$$
\begin{aligned}
\dot{S} & =\int_{a}^{b} \frac{\partial s}{\partial t} d z \\
& =\int_{a}^{b}\left(\mathbf{R}_{\mathbf{0}}(\mathbf{x})^{\top} G_{0}^{\top} \frac{\delta H}{\delta x}+\mathbf{R}_{\mathbf{1}}(\mathbf{x})^{\top} G_{1}^{\top} \frac{\partial}{\partial z} \frac{\delta H}{\delta x}+\right. \\
& \left.g_{s} \mathbf{r}_{\mathbf{s}}(\mathbf{x}) \frac{\partial}{\partial z} \frac{\delta H}{\delta s}+\frac{\partial}{\partial z}\left(g_{s} \mathbf{r}_{\mathbf{s}}(\mathbf{x}) \frac{\delta H}{\delta x}\right)\right) d z
\end{aligned}
$$

The first three terms define the internal entropy production related to the operators of order zero and one

$$
\begin{aligned}
\mathbf{R}_{\mathbf{0}}(\mathbf{x})^{\top} G_{0}^{\top} \frac{\delta H}{\delta x} & =\sum_{i}^{m}\left(R_{0, i}(x) G_{0}(:, i)^{\top} \frac{\delta H}{\delta x}\right) \\
& =\sum_{i}^{m} \gamma_{0, i}\left\{S\left|G_{0}(:, i)\right| H\right\}^{2}=\sum_{i}^{m} \sigma_{0 i} \geq 0, \\
\mathbf{R}_{\mathbf{1}}(\mathbf{x})^{\top} G_{1}^{\top} \frac{\partial}{\partial z} \frac{\delta H}{\delta x} & =\sum_{i}^{m}\left(R_{1, i}(x) G_{1}(:, i)^{\top} \frac{\partial}{\partial z} \frac{\delta H}{\delta x}\right) \\
& =\sum_{i}^{m} \gamma_{i}\left\{S\left|G_{1}(:, i) \frac{\partial}{\partial z}\right| H\right\}^{2}=\sum_{i}^{m} \sigma_{1 i} \geq 0, \\
g_{s} \mathbf{r}_{\mathbf{s}}(\mathbf{x}) \frac{\partial}{\partial z} \frac{\delta H}{\delta s} & =\gamma_{s}\{S \mid H\}^{2}=\sigma_{s} \geq 0,
\end{aligned}
$$

where $\sigma_{0 i}$ and $\sigma_{1 i}$ are, respectively, the zero and first order internal entropy productions due to the $i$-th irreversible thermodynamic processes, and $\sigma_{s}$ is the internal entropy production due to entropy (heat) flux. Since the total internal entropy production is the sum of the internal entropy production of all irreversible processes $\sigma_{t}=\sum_{i}^{m}\left(\sigma_{0 i}+\sigma_{1 i}+\sigma_{s}\right)$ we have

$$
\begin{aligned}
\dot{S} & =\int_{a}^{b} \sigma_{t} d z+\int_{a}^{b} \frac{\partial}{\partial z}\left(g_{s} \mathbf{r}_{\mathbf{s}}(\mathbf{x}) \frac{\delta H}{\delta x}\right) d z \\
& =\int_{a}^{b} \sigma_{t} d z+\left(g_{s} \mathbf{r}_{\mathbf{s}}(\mathbf{x}) \frac{\delta H}{\delta x}(b, t)-g_{s} \mathbf{r}_{\mathbf{s}}(\mathbf{x}) \frac{\delta H}{\delta x}(a, t)\right) \\
& =\int_{a}^{b} \sigma_{t} d z-\left(f_{s}(b, t)-f_{s}(a, t)\right) .
\end{aligned}
$$

from where we have that the supply rate $y_{S}^{\top} v_{s}=$ $\left(f_{s}(b, t)-f_{s}(a, t)\right)$, representing the entropy flux at the boundaries. Hence, the total entropy variation is equal to the internal entropy production minus what is flowing in/out through the boundaries.

\section{Example: the diffusion-reaction process}

Diffusion-reaction processes are systems in which the changes in the mole number per unit volume are due to transport of particles, through processes such as diffusion and convection, and due to chemical reactions. This is the case for instance for tubular reactors (Horn and Jackson, 1972: Feinberg, 1987; Aris, 1989; Kondepudi and Prigogine, 1998; Sandler, 2006). Consider a diffusion-reaction process without convection involving $n$ species and $j$ chemical reactions, described by the following set of PDEs

$$
\begin{gathered}
\frac{\partial c_{i}}{\partial t}=-\frac{\partial f_{c i}}{\partial z}+\sum_{k=1}^{j} \bar{v}_{k i} r_{k}, \quad i=1, \ldots, n \\
\frac{\partial s}{\partial t}=-\frac{\partial f_{s}}{\partial z}+\sum_{k=1}^{n} \sigma_{c_{k}}+\sum_{k=1}^{j} \sigma_{r_{k}}+\sigma_{s} .
\end{gathered}
$$

where $c_{i}$ is the molar concentration per unit volume of the $i$-th specie, $s$ is the entropy density, $f_{i}=-\frac{L_{i}}{T} \frac{\partial \mu_{i}}{\partial z}$ corresponds to the molar flux of the $i$-th specie, $f_{s}=-\frac{\lambda}{T} \frac{\partial T}{\partial z}$ to the entropy flux, $L_{i}$ is the diffusion coefficients of the $i$-th specie, $\lambda$ is heat conduction coefficient, $v_{k i}$ is the stoichiometric coefficient of the reactant $i$ in the $k$ th reaction and $r_{k}$ is the reaction rate of the $k$-th reaction. The thermodynamic driving force of the $k$-th chemical reaction is the chemical affinity of the $k$-th reaction $\mathcal{A}_{k}=-\sum_{i=1}^{n} \bar{v}_{k i} \mu_{i}$, where $\mu_{i}$ is the chemical potential of the $i$-th specie. The internal entropy production of the process is due to irreversible diffusion, heat conduction and to the chemical reactions. In (11) the internal entropy production terms are respectively, $\sigma_{c_{i}}=-\frac{1}{T} f_{c i} \frac{\partial \mu_{i}}{\partial z}=\frac{L_{i}}{T^{2}}\left(\frac{\partial \mu_{i}}{\partial z}\right)^{2}$ for diffusion of the $i$-th 
species, $\sigma_{r_{k}}=\frac{1}{T} r_{k} \mathcal{A}_{k}=\sum_{i=1}^{n-1} \bar{v}_{k i} r_{k} \mu_{i}$ for the $k$-th chemical reaction and $\sigma_{s}=-\frac{1}{T} f_{s} \frac{\partial T}{\partial z}=\frac{\lambda}{T^{2}}\left(\frac{\partial T}{\partial z}\right)^{2}$ for heat conduction.

\subsection{The IPHS model}

Proposition 10. Consider the diffusion-reaction process with $x=\left[c_{1}, \ldots, c_{n}\right]^{\top} \in \mathbb{R}^{n}$. Then (11) can be written as the infinite dimensional IPHS (Definition 1)

$$
\begin{gathered}
\frac{\partial}{\partial t}\left[\begin{array}{c}
x(t, z) \\
s(t, z)
\end{array}\right]=\left[\begin{array}{cc}
0 & G_{0} \mathbf{R}_{\mathbf{0}}(\mathbf{x}) \\
-\mathbf{R}_{\mathbf{0}}(\mathbf{x})^{\top} G_{0}^{\top} & 0
\end{array}\right]\left[\begin{array}{c}
\frac{\partial H}{\delta x}(t, z) \\
\frac{\delta H}{\delta s}(t, z)
\end{array}\right]+ \\
\left.\begin{array}{cc}
0 & \frac{\partial\left(G_{1} \mathbf{R}_{1}(\mathbf{x}) \cdot\right)}{\partial z} \\
\mathbf{R}_{\mathbf{1}}(\mathbf{x})^{\top} G_{1}^{\top} \frac{\partial(\cdot)}{\partial z} & g_{s} \mathbf{r}_{\mathbf{s}}(\mathbf{x}) \frac{\partial(\cdot)}{\partial z}+\frac{\partial\left(g_{s} \mathbf{r}_{\mathbf{s}}(\mathbf{x}) \cdot\right)}{\partial z}
\end{array}\right]\left[\begin{array}{l}
\frac{\delta H}{\delta x}(t, z) \\
\frac{\delta H}{\delta s}(t, z)
\end{array}\right]
\end{gathered}
$$

The modulating function of the mass diffusion of the $i$-th species is

$$
R_{1 i}=\gamma_{1 i}\left\{S\left|G_{1}(:, 1) \frac{\partial}{\partial z}\right| U\right\}=\frac{1}{T}\left(\frac{L_{i}}{T}\right) \frac{\partial \mu_{i}}{\partial z}
$$

with $\gamma_{c i}=\frac{1}{T}\left(\frac{L_{i}}{T}\right)>0,\left\{S\left|G_{1}(:, i) \frac{\partial}{\partial z}\right| U\right\}=\frac{\partial \mu_{i}}{\partial z}$ and the matrices of the operators of order one $G_{1}=I_{n}$. The modulating function of the heat diffusion is

$$
r_{s}=\gamma_{s}\{S \mid U\}=\frac{1}{T}\left(\frac{\lambda}{T}\right) \frac{\partial T}{\partial z}
$$

with $\gamma_{s}=\frac{1}{T}\left(\frac{\lambda}{T}\right)>0,\{S \mid U\}=\frac{\partial T}{\partial z}$ and $g_{s}=1$. The modulating function of the $j$-th chemical reaction is

$$
R_{0 j}=\gamma_{0 j}\left\{s\left|G_{0}(:, j)\right| U\right\}=\frac{1}{T}\left(\frac{r_{j}}{\mathcal{A}_{j}}\right) \mathcal{A}_{j}
$$

with $\gamma_{r_{j}}=\frac{1}{T}\left(\frac{r_{j}}{\mathcal{A}_{j}}\right)>0,\left\{S\left|G_{0 j}\right| U\right\}=\mathcal{A}_{j}$ and the matrix of the operator of order zero $G_{0}=I_{n}$.

Furthermore, consider the following set of boundary inputs and outputs, respectively,

$$
v=\left[\begin{array}{c}
\mathbf{f}(t, a) \\
\mathbf{f}(t, b)
\end{array}\right], \quad y=\left[\begin{array}{c}
-\frac{\delta H}{\delta x}(t, b) \\
-\frac{\delta H}{\delta s}(t, b) \\
\frac{\delta H}{\delta x}(t, a) \\
\frac{\delta H}{\delta s}(t, a)
\end{array}\right],
$$

with $\mathbf{f}=\left[f_{c 1}, \ldots, f_{c n}, f_{s}\right]^{\top}$ the vector of fluxes, then (12) with (13) is a BC-IPHS (Definition 4).

Proof. It is straightforward to verify that the pseudobrackets related to mass and heat diffusion correspond to the respective thermodynamic driving forces. The non-linear functions $\gamma_{c i}$ and $\gamma_{s}$ are positive since the mass diffusion coefficients $L_{i}$, the thermal conductivity coefficient $\lambda$ and the temperature are always positive.
For the thermodynamic driving force of the chemical reaction, consider in a first instance only the $j$-th reaction term. The pseudo-bracket for the $j$-th chemical reaction is

$$
\left\{S\left|G_{0 j}\right| U\right\}=\left[\begin{array}{c}
0 \\
\vdots \\
0 \\
1
\end{array}\right]^{\top}\left[\begin{array}{cccc}
0 & \ldots & 0 & \bar{v}_{j 1} \\
0 & \ldots & 0 & \vdots \\
0 & \ldots & 0 & \bar{v}_{j n} \\
-\bar{v}_{j 1} & \ldots & -\bar{v}_{j n} & 0
\end{array}\right]\left[\begin{array}{c}
\mu_{1} \\
\vdots \\
\mu_{n-1} \\
T
\end{array}\right]=\mathcal{A}_{j} .
$$

On the other hand from De Donder's fundamental equation (Prigogine and Defay, 1954) it has been shown in (Ramirez et al., 2013b) that $\gamma_{r_{j}}=\frac{1}{T}\left(\frac{r_{j}}{\mathcal{A}_{j}}\right)>0$. The same applies for the other reactions. Regarding the boundary inputs and outputs, we have that

$$
P_{e}=\left[\begin{array}{cccc}
0 & 0 & I_{n} & 0 \\
0 & 0 & 0 & 1 \\
I_{n} & 0 & 0 & 0 \\
0 & 1 & 0 & 0
\end{array}\right]
$$

since $P_{1}=0, G_{1}=I_{n}$ and $g_{s}=1$. This implies $M_{p}=$ $P_{e p}=M=P_{e}$, hence choosing the parametrization

$$
\Xi_{1}=\frac{1}{\sqrt{2}}\left[\begin{array}{cccc}
0 & 0 & -I_{n} & 0 \\
0 & 0 & 0 & -1 \\
0 & 0 & I_{n} & 0 \\
0 & 0 & 0 & 1
\end{array}\right], \quad \Xi_{2}=\frac{1}{\sqrt{2}}\left[\begin{array}{cccc}
I_{n} & 0 & 0 & 0 \\
0 & 1 & 0 & 0 \\
I_{n} & 0 & 0 & 0 \\
0 & 1 & 0 & 0
\end{array}\right]
$$

we obtain the boundary inputs/outputs (13).

\subsection{A simple application case}

Consider a simple diffusion-reaction process on $z \in$ $[a, b]$ involving only two species and obeying the following reaction scheme

$$
A \stackrel{r}{\longrightarrow} B
$$

This simple reaction involves four irreversible thermodynamic processes, related to the mass diffusion of species $A$ and $B$, the heat diffusion and the chemical reaction. The thermodynamic parameters of the diffusionreaction process are the mass diffusion coefficients $L_{A}$ and $L_{B}$, the thermal conductivity coefficient $\lambda$ and the stoichiometric coefficients $\bar{v}_{A}=-1$ and $\bar{v}_{B}=1$. The state vector is in this case $x=\left[c_{A}, c_{B}\right]^{\top}$ and $s$, and according to Proposition 10 the BC-IPHS formulation of (14) is

$$
\begin{gathered}
\frac{\partial}{\partial t}\left[\begin{array}{c}
c_{A} \\
c_{B} \\
s
\end{array}\right]=\left[\begin{array}{ccc}
0 & 0 & \frac{r}{T^{2}} \\
0 & 0 & -\frac{r}{T^{2}} \\
-\frac{r}{T^{2}} & \frac{r}{T^{2}} & 0
\end{array}\right]\left[\begin{array}{c}
\mu_{A} \\
\mu_{B} \\
T
\end{array}\right]+ \\
{\left[\begin{array}{ccc}
0 & 0 & \frac{\partial}{\partial z}\left(\frac{L_{A}}{T^{2}} \frac{\partial \mu_{A}}{\partial z}(.)\right) \\
0 & 0 & \frac{\partial}{\partial z}\left(\frac{L_{B}}{T^{2}} \frac{\partial \mu_{A}}{\partial z}(.)\right) \\
\frac{L_{A}}{T^{2}} \frac{\partial}{\partial z}(.) & \frac{L_{B}}{T^{2}} \frac{\partial}{\partial z}(.) & \frac{\lambda}{T}\left(\frac{\partial T}{\partial z}\right) \frac{\partial(.)}{\partial z}+\frac{\partial}{\partial z}\left(\frac{\lambda}{T^{2}} \frac{\partial T}{\partial z}(.)\right)
\end{array}\right]\left[\begin{array}{c}
\mu_{A} \\
\mu_{B} \\
T
\end{array}\right]}
\end{gathered}
$$


The conjugated inputs and outputs are, respectively,

$$
v=\left[\begin{array}{c}
\frac{L_{A}}{T} \frac{\partial \mu_{A}}{\partial z}(t, a) \\
\frac{L_{B}}{T} \frac{\partial \mu_{B}}{\partial z}(t, a) \\
\frac{\lambda}{T} \frac{\partial T}{\partial z}(t, a) \\
\frac{L_{A}}{T} \frac{\partial \mu_{A}}{\partial z}(t, b) \\
\frac{L_{B}}{T} \frac{\partial \mu_{B}}{\partial z}(t, b) \\
\frac{\lambda}{T} \frac{\partial T}{\partial z}(t, b)
\end{array}\right], \quad y=\left[\begin{array}{c}
-\mu_{A}(t, b) \\
-\mu_{B}(t, b) \\
-T(t, b) \\
\mu_{A}(t, a) \\
\mu_{B}(t, a) \\
T(t, a)
\end{array}\right]
$$

i.e., the incoming and outgoing flows of matter and entropy evaluated at the boundaries and the intensive variables evaluated at the boundaries. From Lemma 8 the energy balance is given by

$$
\begin{aligned}
\dot{U} & =y^{\top} v \\
& =\left(\frac{L_{A}}{T} \frac{\partial \mu_{A}}{\partial z} \mu_{A}(b)-\frac{L_{A}}{T} \frac{\partial \mu_{A}}{\partial z} \mu_{A}(a)\right) \\
& +\left(\frac{L_{B}}{T} \frac{\partial \mu_{B}}{\partial z} \mu_{B}(b)-\frac{L_{B}}{T} \frac{\partial \mu_{B}}{\partial z} \mu_{B}(a)\right) \\
& +\left(\frac{\lambda}{T} \frac{\partial T}{\partial z} T(b)-\frac{\lambda}{T} \frac{\partial T}{\partial z} T(a)\right)
\end{aligned}
$$

while by Lemma 9 the entropy balance is

$$
\begin{aligned}
\dot{S}= & \\
\int_{a}^{b} \frac{\lambda}{T^{2}}\left(\frac{\partial T}{\partial z}\right)^{2}+\frac{L_{A}}{T^{2}}\left(\frac{\partial \mu_{A}}{\partial z}\right)^{2} & +\frac{L_{B}}{T^{2}}\left(\frac{\partial \mu_{B}}{\partial z}\right)^{2}+\frac{r}{T^{2}} \mathcal{A} d z \\
& +\left(\frac{\lambda}{T} \frac{\partial T}{\partial z}(b)-\frac{\lambda}{T} \frac{\partial T}{\partial z}(a)\right)
\end{aligned}
$$

We observe that the total internal entropy production is

$\sigma=\int_{a}^{b} \frac{\lambda}{T^{2}}\left(\frac{\partial T}{\partial z}\right)^{2}+\frac{L_{A}}{T^{2}}\left(\frac{\partial \mu_{A}}{\partial z}\right)^{2}+\frac{L_{B}}{T^{2}}\left(\frac{\partial \mu_{B}}{\partial z}\right)^{2}+\frac{r}{T^{2}} \mathcal{A} d z$

Furthermore, $\left\{S\left|G_{1}(:, 1) \frac{\partial}{\partial z}\right| U\right\}=\frac{\partial \mu_{A}}{\partial z},\left\{S\left|G_{1}(:, 2) \frac{\partial}{\partial z}\right| U\right\}=$ $\frac{\partial \mu_{B}}{\partial z},\{S \mid U\}=\frac{\partial T}{\partial z}$ and $\left\{S\left|G_{0}(:, i)\right| U\right\}=\mathcal{A}$ correspond, respectively, to the thermodynamic driving forces of mass and heat diffusion and chemical reactions. The diffusion-reaction process is hence given by the composition of the IPHS formulation of the diffusion process and the chemical reaction together with the mass and heat flows.

\section{Conclusion}

Boundary controlled irreversible port-Hamiltonian systems (BC-IPHS) on 1-dimensional spatial domains have been defined (Definition 1 and 4) by extending the formulation of reversible BC-PHS to irreversible thermodynamic systems controlled at the boundaries of their spatial domains. The structure of BC-IPHS has clear physical interpretation, characterizing the coupling between energy storing and energy dissipating elements, furthermore, the irreversible nature of the model is precisely expressed by the thermodynamic driving forces. By extending the definition of boundary port variables of BC-PHS to deal with the dissipative terms, a set of boundary port variables have been defined such that BC-IPHS are passive with respect to a given set of conjugated inputs and outputs. It is interesting to notice that when no irreversible phenomena is present, and thus the entropy coordinate is not considered, Definition 1 and 4 define a BC-PHS. As for finite dimensional IPHS, the first and second principle are satisfied (Lemmas 8 and 9 as a structural property. The proposed formulation has been illustrated on the examples of an isentropic fluid, with and withot dissipation, heat conduction and a diffusion-reaction process. Future work will study the extension of passivity based boundary control design methods to BC-IPHS.

\section{Acknowledgements}

This work has been partially funded by Chilean FONDECYT 1191544 and CONICYT BASAL FB0008 projects, through grants from the European Commision Marie Skodowska-Curie Fellowship, ConFlex ITN Network under reference code 765579 and from the ANR Agency by the EUR EIPHI, and the INFIDHEM project under the reference codes ANR-16-CE92-0028 and ANR-17-EURE-0002 respectively.

\section{References}

Alonso, A.A., Ydstie, B.E., 1996. Process systems, passivity and the second law of thermodynamics. Computers and Chemical Engineering 20, 1119-1124.

Alonso, A.A., Ydstie, B.E., 2001. Stabilization of distributed systems using irreversible thermodynamics. Automatica 37, 1739-1755.

Alonso, A.A., Ydstie, B.E., Banga, J.R., 2002. From irreversible thermodynamics to a robust control theory for distributed process systems. Journal of Process Control 12, 507-517.

Aris, R., 1989. Elementary chemical reactor analysis. Chemical Engineering, Butterworths, Stoneham, USA.

Brayton, R., Moser, J., 1964. A theory of nonlinear networks. i,. Quarterly of Applied Mathematics 22, 1-33.

Brockett, R., 1977. Control theory and analytical mechanics, in: Martin, C., Hermann, R. (Eds.), Geometric Control Theory. Math Sci Press, Brookline, USA, pp. 1-46.

Brogliato, B., Lozano, R., Maschke, B., Egeland, O., 2020. Dissipative Systems Analysis and Control. Communications and Control Engineering Series. 3rd edition ed., Springer Cham. ISBN 978-3030-19419-2.

Callen, H., 1985. Thermodynamics and an introduction to thermostatistics. Wiley, New-York. 
Christofides, P.D., 2001. Nonlinear and robust control of PDE systems: methods and applications to transport-reaction processes. 1 ed., Springer.

Christofides, P.D., Daoutidis, P., 1998. Robust control of hyperbolic pde systems. Chemical Engineering Science 53, 85 - 105.

Cortés, J., van der Schaft, A., Crouch, P.E., 2005. Characterization of gradient control systems. SIAM J. Control Optim. 44, 1192-1214. doi http://dx .doi.org/10.1137/S0363012903425568

Duindam, V., Macchelli, A., Stramigioli, S., Bruyninckx, H. (Eds.), 2009. Modeling and Control of Complex Physical Systems - The Port-Hamiltonian Approach. Springer-Verlag, Berlin, Germany.

Eberard, D., Maschke, B.M., van der Schaft, A.J., 2007. An extension of Hamiltonian systems to the thermodynamic phase space: Towards a geometry of nonreversible processes. Reports on Mathematical Physics 60, 175-198.

Favache, A., Dochain, D., 2010. Power-shaping control of reaction systems: The CSTR case. Automatica 46, 1877 - 1883

Favache, A., Dochain, D., Maschke, B., 2010. An entropy-based formulation of irreversible processes based on contact structures. Chemical Engineering Science 65, 5204-5216.

Favache, A., Dochain, D., Winkin, J., 2011. Power-shaping control: Writing the system dynamics into the Brayton-Moser form. Systems \& Control Letters 60, 618 - 624

Feinberg, M., 1987. Chemical reaction network structure and the stability of complex isothermal reactors-i. the deficiency zero and deficiency one theorems. Chemical Engineering Science 42, 2229 -2268 .

Gay-Balmaz, F., Yoshimura, H., 2018. A variational formulation of nonequilibrium thermodynamics for discrete open systems with mass and heat transfer. Entropy 20 , 163. URL: http://dx.doi.org/10.3390/e20030163. doi $10.3390 / \mathrm{e} 20030163$

Godasi, S., Karakas, A., Palazoglu, A., 2002. Control of nonlinear distributed parameter processes using symmetry groups and invariance conditions. Computers \& Chemical Engineering 26, $1023-$ 1036.

Grmela, M., Öttinger, H., 1997. Dynamics and thermodynamics of complex fluids. I. development of a general formalism. Physical Review E 56, 6620-6632.

Hoang, H., Couenne, F., Jallut, C., Le Gorrec, Y., 2011. The port Hamiltonian approach to modelling and control of continuous stirred tank reactors. Journal of Process Control 21, 1449-1458.

Hoang, H., Couenne, F., Jallut, C., Le Gorrec, Y., 2012. Lyapunovbased control of non isothermal continuous stirred tank reactors using irreversible thermodynamics. Journal of Process Control 22, $412-422$.

Horn, F., Jackson, R., 1972. General mass action kinetics. Archive for Rational Mechanics and Analysis 47, 81-116.

Jacob, B., Zwart, H., 2012. Linear Port-Hamiltonian Systems on Infinite-dimensional Spaces. volume 223 of Operator Theory: Advances and Applications. Birkhäuser, Basel, Switzerland.

Keenan, J.H., 1951. Availability and irreversibility in Thermodynamics. British Journal of Applied Physics 2, 183. URL: http://stacks. iop.org/0508-3443/2/i=7/a=302

Kondepudi, D., Prigogine, I., 1998. Modern Thermodynamics: From Heat Engines to Dissipative Structures. John Wiley \& Sons, Chichester, England.

Le Gorrec, Y., Villegas, J., Zwart, H., Maschke, B., 2006. Dissipative boundary control systems with application to distributed parameters reactors, in: Joint CCA, ISIC and CACSD Conference 2006, Munich, Germany.

Le Gorrec, Y., Zwart, H., Maschke, B., 2005. Dirac structures and boundary control systems associated with skew-symmetric differential operators. SIAM Journal on Control and Optimization 44, 1864-1892.
Macchelli, A., Le Gorrec, Y., Ramirez, H., 2020. Exponential stabilisation of port-hamiltonian boundary control systems via energyshaping. IEEE Transactions on Automatic Control , 1-1.

Macchelli, A., Le Gorrec, Y., Ramirez, H., Zwart, H., 2017. On the synthesis of boundary control laws for distributed portHamiltonian systems. IEEE Transactions on Automatic Control 62, 1700-1713.

Marsden, J., 1992. Lectures on Mechanics. Number 174 in London Mathematical Socitety Lecture Notes Series, Cambridge University Press, Cambridge, New York, USA.

Maschke, B., van der Schaft, A., 1992. Port controlled Hamiltonian systems: modeling origins and system theoretic properties, in: Proceedings of the 3rd IFAC Symposium on Nonlinear Control Systems, NOLCOS'92, Bordeaux, France. pp. 282-288.

Maschke, B., van der Schaft, A., 2005. Advanced Topics in Control Systems Theory: Lecture Notes from FAP 2004. Springer London, London. chapter 4 Compositional Modelling of DistributedParameter Systems. pp. 115-154.

Merker, J., Krüger, M., 2013. On a variational principle in thermodynamics. Continuum Mechanics and Thermodynamics 25, 779793. doi 10.1007/s00161-012-0277-2

Mrugala, R., Nulton, J., Schon, J., Salamon, P., 1991. Contact structure in thermodynamic theory. Reports in Mathematical Physics 29, 109-121.

Mushik, W., Gümbel, S. and Kröger, M., Öttinger, 2000. A simple example for comparing GENERIC with rational non-equilibrium Thermodynamics. Physica A 285, 448-466.

Nijmeijer, H., van der Schaft, A., 1990. Nonlinear Dynamical Control Systems. Springer-Verlag, New York, USA.

Olver, P., 1993. Application of Lie groups to differential equations. Springer-Verlag.

Ortega, R., Loria, A., Nicklasson, P., Sira-Ramirez, H., 1998. Passivity-based control of Euler-Lagrange Systems. Communications and Control Series, Springer, Berlin.

Öttinger, H., Grmela, M., 1997. Dynamics and thermodynamics of complex fluids. II. illustrations of a general formalism. Physical Review E 56, 6633-6655.

Prigogine, I., Defay, R., 1954. Chemical Thermodynamics. volume 1 of Treatise on Thermodynamics. Longmans Green and Co, London, Great Britain.

Ramirez, H., Le Gorrec, Y., 2016. An irreversible portHamiltonian formulation of distributed diffusion processes. IFACPapersOnLine 49, 46 - 51. 2th IFAC Workshop on Thermodynamic Foundations for a Mathematical Systems Theory TFMST 2016.

Ramirez, H., Le Gorrec, Y., Macchelli, A., Zwart, H., 2014a. Exponential stabilization of boundary controlled port-Hamiltonian systems with dynamic feedback. Automatic Control, IEEE Transactions on PP, 1-1. doi 10.1109/TAC.2014.2315754

Ramirez, H., Le Gorrec, Y., Maschke, B., Couenne, F., 2016. On the passivity based control of irreversible processes: A portHamiltonian approach. Automatica 64, $105-111$.

Ramirez, H., Maschke, B., Sbarbaro, D., 2013a. Feedback equivalence of input-output contact systems. Systems \& Control Letters $62,475-481$.

Ramirez, H., Maschke, B., Sbarbaro, D., 2013b. Irreversible portHamiltonian systems: A general formulation of irreversible processes with application to the CSTR. Chemical Engineering Science $89,223-234$

Ramirez, H., Maschke, B., Sbarbaro, D., 2013c. Modelling and control of multi-energy systems: An irreversible port-Hamiltonian approach. European Journal of Control 19, 513 - 520.

Ramirez, H., Maschke, B., Sbarbaro, D., 2017a. Partial stabilization of input-output contact systems on a Legendre submanifold. IEEE Transactions on Automatic Control 62, 1431-1437. 
Ramirez, H., Sbarbaro, D., Maschke, B., 2014b. Irreversible portHamiltonian formulation of chemical reaction networks, in: The 21st International Symposium on Mathematical Theory of Networks and Systems, Groningen, The Netherlands.

Ramirez., H., Sbarbaro, D., Ortega, R., 2009. On the control of nonlinear processes: An IDA-PBC approach. Journal of Process Control 19, $405-414$

Ramirez, H., Zwart, H., Le Gorrec, Y., 2017b. Stabilization of infinite dimensional port-Hamiltonian systems by nonlinear dynamic boundary control. Automatica 85, 61 - 69 .

Sandler, S., 2006. Chemical, Biochemical, and Engineering Thermodynamics. Fourth ed., John Wiley \& Sons, Hoboken, USA.

van der Schaft, A., 1986. On feedback control of Hamiltonian systems, in: Byrnes, C.I., Lindquist, A. (Eds.), Theory and Applications of Nonlinear Control Systems. Elsevier North-Holland, New York, USA, pp. 273-290.

van der Schaft, A., Jeltsema, D., 2014. Port-Hamiltonian systems theory: An introductory overview. Foundations and Trends in Systems and Control 1, 173-378. URL: $\quad$ http://dx.doi.org/10.1561/2600000002 doi $10.1561 / 2600000002$

van der Schaft, A., Maschke, B., 1995. The Hamiltonian formulation of energy conserving physical systems with external ports. Archiv für Elektronik und Übertragungstechnik 49, 362-371.

van der Schaft, A., Maschke, B., 2002. Hamiltonian formulation of distributed-parameter systems with boundary energy flow. Journal of Geometry and Physics 42, 166 - 194.

van der Schaft, A., Maschke, B., 2018. Geometry of thermodynamic processes. Entropy 20, 925.

van der Schaft, A.J., 2000. L2-Gain and Passivity Techniques in Nonlinear Control.

Schaum, A., Meurer, T., Moreno, J.A., 2018. Dissipative observers for coupled diffusion-convection-reaction systems. Automatica $94,307-314$.

Smale, S., 1972. On the mathematical foundations of electrical circuit theory. J. of Differential Geometry 7, 193-210.

Willems, J., 1972. Dissipative dynamical systems part I: General theory. Archive for Rational Mechanics and Analysis 45, 321-351.

\section{Appendix A. Port-Hamiltonian systems on infinite dimensional spaces}

Let us now define a port-Hamiltonian system on an infinite dimensional space. To this end, first we introduce the definition of variational derivative of a functional, see Nijmeijer and van der Schaft (1990).

Definition 11. Consider a functional $H$ defined by

$$
H(x)=\int_{a}^{b} h\left(z, x, x^{(1)}, \ldots, x^{(n)}\right) d z
$$

for any smooth real vector function $x(z), z \in Z=(a, b)$ where the integrand $u$ is a smooth function of $x$ and its derivatives up to some order $n$. The variational derivative of the functional $H$ is denoted by $\frac{\delta H}{\delta x}$ and is the only function that satisfy for every $\epsilon \in \mathbb{R}$ and smooth real function $\delta x(z), z \in Z$, such that their derivatives satisfy $\delta x^{(i)}(a)=\delta x^{(i)}(b)=0, i=0, \ldots, n$,

$$
H[x+\epsilon \delta x]=H[x]+\epsilon \int_{a}^{b} \frac{\delta H}{\delta x} \delta x d z+O\left(\epsilon^{2}\right)
$$

In the case when $h$ only depends on the vector function $x$ and not its derivatives, then the variational derivative is simply obtained by derivation of the integrand, i.e,

$$
\frac{\delta H}{\delta x}=\frac{\partial h}{\partial x} .
$$

An infinite dimensional PHS on a 1D spatial domain is characterized by the following PDE

$$
\frac{\partial x}{\partial t}(t, z)=P_{1} \frac{\partial}{\partial z}\left(\frac{\delta H}{\delta x}(t, z)\right)+\left(P_{0}-G_{0}\right) \frac{\delta H}{\delta x}(t, z),
$$

with $z \in(a, b), P_{1} \in M_{n}(\mathbb{R})^{3}$ a nonsingular symmetric matrix, $P_{0}=-P_{0}^{\top} \in M_{n}(\mathbb{R}), G_{0} \in M_{n}(\mathbb{R})$ with $G_{0} \geq 0$ and $x$ taking values in $\mathbb{R}^{n}$. The controlled (and homogeneous) boundary conditions of A.1 are characterized by a matrix $W_{B}$ of appropriate size such that

$$
v(t)=W_{B}\left[\begin{array}{l}
\frac{\delta H}{\delta x}(t, b) \\
\frac{\delta H}{\delta x}(t, a)
\end{array}\right]
$$

Considering the above boundary conditions as the input of the system, we can define an associate boundary output as

$$
y(t)=W_{C}\left[\begin{array}{l}
\frac{\delta H}{\delta x}(t, b) \\
\frac{\delta H}{\delta x}(t, a)
\end{array}\right] .
$$

If $W_{B}$ and $W_{C}$ satisfy

$$
\begin{aligned}
& W_{B} \tilde{\Sigma} W_{B}^{\top}=W_{C} \tilde{\Sigma} W_{C}^{\top}=0 \\
& W_{B} \tilde{\Sigma} W_{C}^{\top}=W_{C} \tilde{\Sigma} W_{B}^{\top}=I
\end{aligned}
$$

with $\tilde{\Sigma}=\left[\begin{array}{cc}P_{1}^{-1} & 0 \\ 0 & -P_{1}^{-1}\end{array}\right]$, it is not difficult to show that under some very general conditions (Le Gorrec et al., 2005; Jacob and Zwart, 2012) the change of energy of the system becomes

$$
\dot{H}(t)=y^{\top}(t) v(t)-\int_{a}^{b} \frac{\delta H^{\top}}{\delta x}(t, z) G_{0} \frac{\delta H}{\delta x}(t, z) d z
$$

We can see from this equation that the dissipation in the system is characterized by the matrix $G_{0}$. Indeed, since the input and output act and sense at the boundary of the spatial domain, in the absence of internal dissipation $\left(G_{0}=0\right)$ the system only exchanges energy with the environment through the boundaries. In this case the PHS fullfils

$$
\dot{H}(t)=y^{\top}(t) v(t),
$$

and the PHS is called conservative. This formulation has proven to be extremely useful to study the

\footnotetext{
${ }^{3} M_{n}(\mathbb{R})$ denote the space of real $n \times n$ matrices
} 
existence and uniqueness of solutions for the linear case, and to perform control synthesis for the general class of PHS (Le Gorrec et al.,, 2005; Jacob and Zwart, 2012; Ramirez et al., 2014a; Macchelli et al., 2017; Ramirez et al., 2017b). One interesting feature of PHS is that they are applicable to hyperbolic and parabolic systems, however the PHS formulation of parabolic systems leads necessary to an implicit system. 